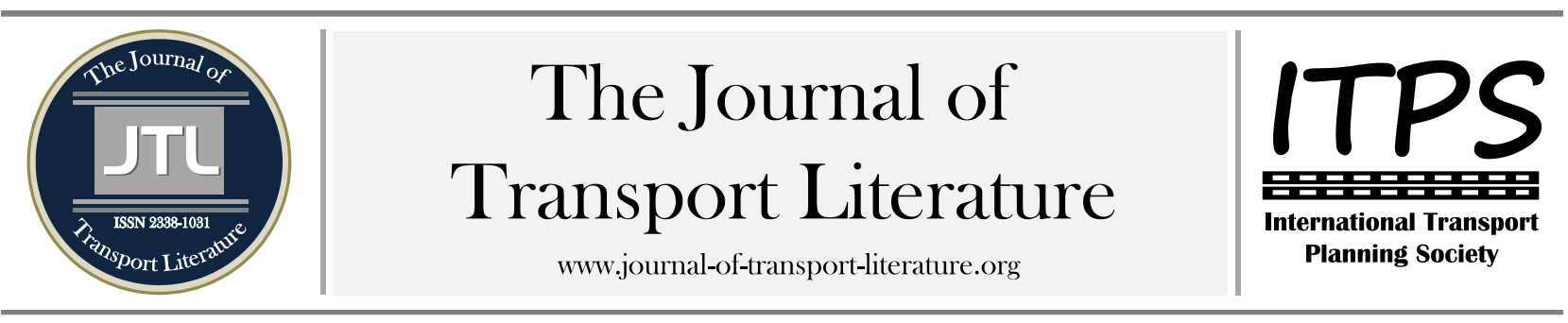

\title{
Procedimento para uso de Redes Neurais Artificiais no planejamento estratégico de fluxo de carga no Brasil
}

Daniel Neves Schmitz Gonçalves ${ }^{1,+}$; Marcelino Aurélio da Silva²; Márcio de Almeida d'Agosto²

${ }^{1}$ Military Institute of Engineering, Rio de Janeiro, Brazil

${ }^{2}$ Federal University of Rio de Janeiro, Rio de Janeiro, Brazil

\section{Article Info}

Palavras-chave:

redes neurais

transporte de soja

previsão de viagens de carga

distribuição de viagens

Submitted 15 Mar 2014;

received in revised form 5 Apr 2014;

accepted 8 Oct 2014.

Licensed under

Creative Commons

CC-BY $3.0 \mathrm{BR}$.

\section{Resumo}

0 presente trabalho tem por finalidade utilizar a técnica de Redes Neurais Artificiais (RNA) para estimar a matriz origem-destino (O-D) de grãos de soja no Brasil destinado à exportação a fim de explicar a variabilidade dos fluxos entre os pares O-D, considerando as características dinâmicas existentes nas matrizes $\mathrm{O}-\mathrm{D}$, comparando os resultados com o modelo gravitacional (MG), que é um modelo utilizado em planejamentos estratégicos do governo brasileiro, propondo também um procedimento para a utilização das RNAs na distribuição de viagens de carga. Dentre os 4 modelos construídos neste artigo, destacou-se a combinação C-02, apresentando um coeficiente de determinação $\left(R^{2}\right)$ superior em $9 \%$ e tendo Índice de Dissimilaridade (ID) 6,92\% menor em relação ao MG. Pode-se observar que as RNAs podem ser um substituto potencial aos modelos estatísticos convencionais, por sua fácil interface dos programas com o usuário, porém a mesma não consta na literatura como uma opção para o cálculo da distribuição de viagens do método sequencial de quatro etapas e esta técnica pouco foi aplicada para o transporte de carga.

+ Corresponding author. Instituto Militar de Engenharia. Praça General Tibúrcio, 80 - Praia Vermelha - Urca - 22290-270 Rio de Janeiro - RJ - Brasil.

E-mail address: daniel.schmitz.jf@gmail.com.

\section{Introdução}

Este trabalho visa desenvolver um procedimento de análise e previsão da demanda e oferta de soja no Brasil para exportação, sendo os pontos de origem as mesorregiões produtoras e os destinos os portos brasileiros. Como hipótese do trabalho, definiu-se que é possível utilizar redes neurais artificiais (RNAs) como um dos modelos de distribuição de viagens, correspondente a segunda etapa do Método de quatro etapas. Como premissa do trabalho, definiu-se que é possível utilizar o método de quatro etapas no planejamento de transporte de carga, com finalidade de estimar os fluxos de carga na região de estudo.

A análise e a previsão de demanda de transporte de carga é um estudo necessário para se levantar as prioridades de investimentos no sistema de transporte de uma região, sendo uma ferramenta útil para auxiliar o planejamento estratégico. Porém, a explicação do comportamento dos fluxos de cargas entre as regiões não é simples, necessitando de considerações e ferramentas adequadas para sua previsão. Um modelo utilizado para a análise e a previsão de viagens é o Modelo Gravitacional (MG) sendo este utilizado no Plano Nacional de Logística e Transportes (PNLT), Plano Nacional de Integração Hidroviária (PNIH), para estimar a demanda de passageiros e carga e pelo Departamento Nacional de Estradas de Rodagem (DNER) e o Ministério dos transportes para o cálculo da estimativa de demanda nas licitações de serviços de transporte interestadual de passageiros, logo seria de grande valia, analisar outro modelo, para termos uma nova opção.

Embora não esteja evidenciado na literatura, as RNA podem ser utilizadas na segunda e terceira etapa do Método sequencial de quatro etapas. Segundo Brondino (1999), nos problemas de previsão a modelagem através de RNA surge como um substituto potencial aos modelos estatísticos convencionais, pela fácil interface dos programas com o usuário e a não necessidade de possuir conhecimento prévio da relação das variáveis envolvidas, ao contrário de modelos estatísticos utilizados para o mesmo fim. Logo, as RNAs têm sido amplamente utilizadas como técnica de suporte ao planejamento de transportes (Alves; Silva; Waerden, 2012). De acordo Souza e D' Agosto (2012) existe uma lacuna na publicação de trabalhos sobre o uso do método de quatro etapas como ferramenta de planejamento de transporte de cargas no Brasil, em periódicos científicos, correspondendo apenas 9\% dos trabalhos levantados e segundo Corrêa (2008) poucos são os trabalhos realizados com a utilização da técnica de RNAs na área de transporte de cargas e logística, sendo a maioria deles aplicados ao transporte de passageiros. Logo este trabalho tem como objetivo geral, mostrar a aplicação da RNA na segunda etapa do Método sequencial e criar um procedimento padrão para a utilização desta no transporte de carga.

Em 2010, as exportações brasileiras de soja em grão, farelo de soja e óleo de soja foram avaliados em US \$ 10 bilhões, representando $27 \%$ do total das exportações agrícolas do país (Intercâmbio Comercial do Agronegócio, 2011). Dos 264 milhões de toneladas de soja produzido no mundo na safra 2010-11, o Brasil foi responsável por 75 milhões, ou $28 \%$ da 
produção global, tornando-se o segundo maior produtor de soja, atrás apenas dos Estados Unidos, que produziu 91 milhões de toneladas, ou seja, 21\% a mais do que o Brasil (EMBRAPA, 2011).

Devido às características de clima, fertilidade do solo e dos preços da terra, as áreas de produção de soja no Brasil estão concentradas em lugares distantes de áreas de consumo e portos de exportação (Scalea, 2002). Esta situação requer um sistema de transporte eficiente para minimizar os custos e aumentar a competitividade dos produtos de soja brasileiros no mercado mundial. A formulação de planejamentos estratégicos para tal sistema de transporte requer o conhecimento do comportamento da demanda para este serviço ao longo do tempo para permitir a projeção de demanda e alternativas de investimento em oferta de transporte (Bruton, 1979).

No trabalho de da Silva e D'Agosto (2013), uma matriz origem-destino (O-D) de soja no Brasil foi estimada utilizando-se o MG tradicional com uma característica de separar a modelagem em dois estágios. 0 primeiro estágio refere-se a tendência observada dos dados para estimar um comportamento futuro já o segundo estágio trata do balanceamento da matriz 0-D, melhorando as medidas de desempenhos analisadas. Neste trabalho, no lugar de se utilizar o modelo gravitacional para explicar a tendência será utilizado uma RNA com as mesmas variáveis do MG tradicional a fim de explicar a tendência observada. Logo, este artigo propõe um modelo para estimar a matriz 0-D de grãos de soja no Brasil destinado à exportação com aplicação de RNA para o estágio de tendência e programação multiobjetivo para o estágio de balanceamento, o mesmo apresentou uma precisão consideravelmente maior do que o MG, mostrando ser uma nova e confiável opção

\section{Redes Neurais Artificiais}

As RNAs são modelos matemáticos que tentam simular o funcionamento do cérebro humano com objetivo de resolver problemas da vida real. As RNAs são utilizadas nas mais diversas áreas de aplicação como previsão, classificação, controle entre outros (Brondino, 1999). Pode-se afirmar que RNAs são sistemas paralelos distribuídos, compostos por unidades de processamento simples (nós) que calculam determinadas funções matemáticas, que normalmente não são lineares (Alves; Silva; Waerden, 2012). As redes neurais têm um potencial de aplicação muito vasto na área de transportes, Madalozo e Dougherty (1997), citam como exemplo destas aplicações: em estudos de comportamento de motoristas de veículos autônomos, estimação de parâmetros em pesquisas de fluxo de tráfego, manutenção de pavimentos, classificação/detecção de veículos, análise de modelos de tráfego, operações de transporte de carga, previsão de tráfego, política e economia de transporte, transporte aéreo, transporte marítimo, veículos submarinos, operação de metrôs e controle de tráfego. De acordo com Raia Jr (2000), um dos primeiros estudos de aplicação de RNAs na área de Engenharia de Transporte teria sido o de Nakatsuji e Kaku (1989), procurando resolver problemas relacionados com a Engenharia de Tráfego. Conforme Corrêa (2008) desde os anos de 1980, a técnica de RNA vem sendo utilizada nos países desenvolvidos. Já no Brasil, isto aconteceu a partir da segunda metade da década de 1990 e, desde então, diversos trabalhos vêm sendo desenvolvidos com a aplicação das RNAs, particularmente com aplicações no Setor de Transportes.

A fim de ampliar o conhecimento da aplicação de RNA e criar um procedimento, foram analisados diversos trabalhos que utilizaram RNAs com aplicação no planejamento de transportes, especificamente quanto a previsão de demanda/viagens, destes trabalhos, podemos destacar: Júnior (2004); Raia Jr (2000); Junior, Moreira, Pessanha e Jacinto (2004); Celik (2004); Madalozo, Dyminski e Ribeiro (2004), Dia (2001); Kirby, Watson e Dougherty (1997) e Dougherty e Cobbett (1997). Onde foram identificados os softwares utilizados, objetivos, número de neurônios na camada intermediária, função de normalização, variáveis de entrada, número de camadas ocultas, algoritmos de aprendizagem utilizados e a proporção dos dados utilizados no treinamento, validação e teste.

\section{Procedimento proposto}

O fluxograma mostrado na Figura 1 mostra as cinco etapas do procedimento proposto neste artigo com base na revisão bibliográfica e nos artigos analisados, para estimar matrizes O-D de carga. Os próximos subitens desta seção apresentarão os detalhes de cada etapa com os resultados para aplicação do caso de exportação de soja no Brasil. 0 treinamento da RNA e as estimativas foram feitas por meio do software MathWorks MatLABC que além de oferecer um ambiente completamente amigável, admite a utilização de ferramentas úteis ao desenvolvimento intuitivo de Redes Neurais (Araujo e Melo, 2006).

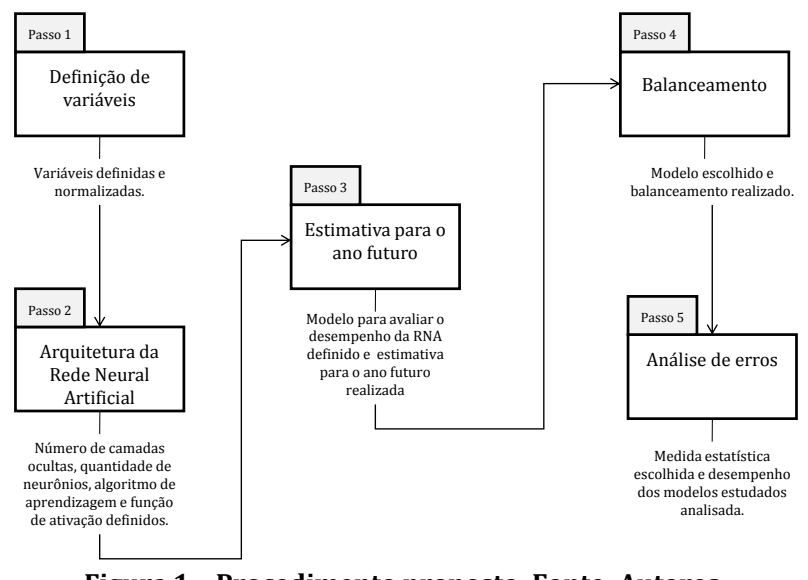

Figura 1 - Procedimento proposto. Fonte: Autores.

\subsection{Definição das variáveis}

Na primeira etapa deverão ser definidas as variáveis independentes que serão utilizadas como entrada para a RNA. Por se tratar de estimativas de matrizes O-D de fluxos de carga tentou-se neste trabalho buscar uma analogia com os modelos gravitacionais e entrópicos. Para esses modelos as variáveis independentes são: Oi que representa a quantidade de carga em toneladas que saem de cada zona de tráfego de origem i; Dj a quantidade de carga em toneladas que chega em cada zona de 
tráfego j; e dij é a impedância ente a origem i e o destino j. A variável dependente é o Tij que é a quantidade de carga em tonelada que sai da origem i para o destino j. A impedância é qualquer variável que quanto maior o seu valor diminui a probabilidade de ocorrer viagens ou fluxos de carga ou pessoas como: distância, tempo, valor do frete, custos logísticos entre outros (Ortuzar; Willumsen, 2011). Os valores dos fluxos (Tij), para aplicação neste artigo, foram obtidos no portal ALICE_WEB (2013). Os valores referem-se ao fluxo de soja destinada à exportação em toneladas. Os fluxos foram disponibilizados por mesorregiões de origem até o porto de destino para os anos de 2002 até 2010, com isso foi possível montar a matriz O-D para cada ano. A impedância adotada na aplicação foi à distância rodoviária, uma vez que o modo rodoviário representa mais de 61,1\% do transporte de carga no Brasil (CNT, 2012) e os dados obtidos não estão separados por modos de transportes. As distâncias rodoviárias foram determinadas com o auxílio do software TransCADC versão 5.0. A escolha do TransCAD (C), se deu pelo fato do mesmo entre os diversos SIG's existentes, tem se destacado, sendo conhecido na literatura como sendo um SIG especialmente voltado para transportes (Kaiser et al., 2007).

\subsection{Arquitetura da RNA}

Definida a topologia da RNA composta por uma camada de saída; uma de entrada; e uma ou mais ocultas, deve-se determinar a quantidade de camadas ocultas e a quantidade de neurônios em cada uma delas bem como o algoritmo de aprendizado e a função de ativação que serão utilizados. Na aplicação da RNA para o problema deste artigo, decidiu-se avaliar o comportamento da RNA para uma camada oculta, utilizando como função de ativação a tangente hiperbólica. Testes ainda preliminares não mostraram ganho de desempenho com mais de uma camada oculta e com outras funções de ativação e Batista (2012) ressalta que o aumento do número de camadas acarreta o aumento da complexidade e do tempo de processamento da rede. Segundo Aguiar Júnior (2004) a melhor opção para função de ativação dos neurônios, tanto na camada intermediária quanto na camada de saída, foi a função da tangente hiperbólica. Dentre diversas variações do algoritmo Backpropagation, foi utilizado o algoritmo de aprendizagem Levenberg-Marquardt (LM), que segundo Araújo e Melo (2006) e Yuehua et al (2008) caracteriza-se como um dos métodos mais rápidos para o treinamento de RN Acíclicas de tamanho moderado, além disso é eficaz na minimização de funções com um pequeno número de parâmetros e o tempo de convergência da rede tende a ser menor quando comparado aos outros modelos e possui elevada precisão.

Para realizar a análise do comportamento da RNA foram definidas duas combinações de entradas e saídas de dados conforme a Tabela 1.

Tabela 1 - Combinações avaliadas. Fonte: Autores.

\begin{tabular}{lll}
\hline Combinação & Dados de entrada & Dados de saída \\
\hline C-01 & Oi, Dj e dij & Tij \\
C-02 & Ln Oi, Ln Dj e Ln dij & Ln Tij \\
\hline
\end{tabular}

Os dados obtidos foram divididos em 4 conjuntos. A primeira divisão foi a retirada do ano de 2010 com objetivo de estimá-lo no final de todo treinamento da RNA. Depois disso, todos os dados de 2002 a 2009 foram divididos aleatoriamente em 70\% para treinamento, 15\% para teste e 15\% para validação. Não existe uma formula ou método na literatura que possa auxiliar na identificação do número de neurônios na camada oculta para o conjunto de treinamento. Para estipular este número, testou-se um intervalo de 1 à 15 neurônios avaliando seus respectivos $\mathrm{R}^{2} .0$ valor do $\mathrm{R}^{2}$ refere-se ao ponto em que se deu a parada do treinamento. Para o C-01 o maior $\mathrm{R}^{2}$ obtido foi utilizando 10 neurônios e para C-02, 7. Para quantidade de neurônios maiores não houve ganho no valor de $\mathrm{R}^{2}$.

\subsection{Estimativa para o ano futuro}

Nesta etapa, deverão ser realizadas as estimativas para o ano futuro com a RNA definida no passo anterior. Com objetivo de avaliar o desempenho da RNA definida do artigo, foi definido um modelo gravitacional. A Eq. 1 mostra o modelo utilizado por da Silva e D'Agosto (2013). Os valores de $\chi_{i} e \psi_{j}$ serão determinados na etapa 4, pois estes são os coeficiente de balanceamento. 0 modelo de tendência é definido adotando os valores do coeficiente de balanceamento iguais a 1 e encontrando os parâmetros $\alpha, \beta, \gamma$ e pelo método dos mínimos quadrados ordinários.

$$
T_{i j}=\alpha \cdot \chi_{i} \cdot \psi_{j} \cdot \frac{O_{i}^{\beta} \cdot D_{j}^{\gamma}}{d_{i j}^{\delta}}
$$

\subsection{Balanceamento}

Para um período futuro em que se deseja estabelecer a matriz 0-D, estima-se os valores de fluxos baseado na tendência observada e determinada no passo 4 e, em seguida, determina-se os coeficientes de balanceamento $\left(\chi_{i}\right.$ e $\left.\psi_{j}\right)$ por meio da formulação de um problema de otimização de multi-objetivos proposto por da Silva e D'Agosto (2013), uma vez que se tem como objetivo atender todas as restrições apresentadas na Tabela 2. A solução adotada para modelagem foi a formulação de modelo por programação por metas, utilizando o método dos pesos (da Silva; D’Agosto, 2013).

Tabela 2 - Função objetivo e restrições para o balanceamento da matriz 0-D

Fonte: Silva e D'Agosto, 2013

\begin{tabular}{lcc}
\hline Restrição & Função objetivo & $\begin{array}{l}\text { Função objetivo ponderada } \\
\text { (Minimizar) }\end{array}$ \\
\hline$\sum_{j} T_{i j}=O_{i}$ e & Minimizar $\begin{cases}a_{j}^{+}+a_{j}^{-}, & j=1,2,3, \ldots, J \\
\sum_{i}^{+}+p_{i}^{-}, & i=1,2,3, \ldots, I\end{cases}$ & $\sum_{i=1}^{I} \eta_{i}\left(p_{i}^{+}+p_{i}^{-}\right)+\sum_{j=1}^{J} \vartheta_{j} \cdot\left(a_{j}^{+}+a_{j}^{-}\right)$ \\
\hline
\end{tabular}

As metas podem ser expressas da seguinte maneira:

$\sum_{i=1}^{I} T_{i j}-a_{j}^{+}+a_{j}^{-}=D_{j}$, para todo j.

$\sum_{j=1}^{J} T_{i j}-p_{i}^{+}+p_{i}^{-}=O_{i}$, para todo i.

$A_{i} . B_{j}$, Tij, $a_{j}^{+}, a_{j}^{-}, p_{i}^{+}$e $p_{i}^{-} \geq 0$, para todo i e j.

Sendo: $\mathrm{a}_{\mathrm{j}}^{+}$: desvio positivo do $\sum_{\mathrm{i}=1}^{\mathrm{I}} \mathrm{T}_{\mathrm{ij}}$ em relação à $\mathrm{D}_{\mathrm{j}}$, para cada destino $\mathrm{j}$; 
$\mathrm{a}_{\mathrm{j}}^{-}$: desvio negativo do $\sum_{\mathrm{i}=1}^{\mathrm{I}} \mathrm{T}_{\mathrm{ij}}$ em relação à $\mathrm{D}_{\mathrm{j}}$, para cada destino $\mathrm{j}$;

$\mathrm{p}_{\mathrm{i}}^{+}$: desvio positivo do $\sum_{\mathrm{j}=1}^{\mathrm{J}} \mathrm{T}_{\mathrm{ij}}$ em relação à $\mathrm{O}_{\mathrm{i}}$, para cada origem $\mathrm{i}$.

$\mathrm{p}_{\mathrm{i}}^{-}$: desvio negativo do $\sum_{\mathrm{j}=1}^{\mathrm{J}} \mathrm{T}_{\mathrm{ij}}$ em relação à $\mathrm{O}_{\mathrm{i}}$, para cada origem $\mathrm{i}$.

$\eta_{\mathrm{i}}$ : peso do desvio ocorrido em relação à produção da origem $\mathrm{i}$;

$\vartheta_{\mathrm{j}}$ : peso do desvio ocorrido em relação à atração do destino $\mathrm{j}$.

Para encontrar os valores dos coeficientes de balanceamento foi utilizado o software Premium Solver Pro@ versão educacional. A busca pelos valores de $\chi_{i} e \psi_{j}$ é um problema de otimização não-linear e o "solver@" utiliza o método do Gradiente Reduzido Generalizado (GRG) para resolver. Devido a quantidade excessiva de coeficientes de balanceamento será apresentado apenas os valores de desempenho da Tabela 3 como resultado final do modelo.

\section{Análise de erros}

Uma importante componente na construção de um modelo é a avaliação da capacidade de replicar um conjunto de dados conhecidos. Para avaliar esta capacidade, medidas estatísticas, conhecidas como goodness-of-fit, são utilizadas com objetivo de medir a proximidade entre o conjunto observado e o estimado pelo modelo, como pode ser visto nos trabalhos de Wilson (1970), Wilson (1976), Smith e Hutchinson (1981), Knudsen e Fotheringham (1986) e Sikdar e Hutchinson (1981). As medidas que mais se destacaram nos trabalhos citados anteriormente foram o Índice de Dissimilaridade (ID) e coeficiente de determinação (R2). A Tabela 3 apresenta os valores das goodness-of-fit para todos os modelos estudados, aplicados na estimativa do ano de 2010.

Tabela 3 - Análise de desempenho dos modelos estudados. Fonte: Autores

\begin{tabular}{|c|c|c|c|c|c|c|}
\hline \multirow{4}{*}{ goodness-of-fit } & \multicolumn{4}{|c|}{$\begin{array}{l}\text { Modelo com RNA } \\
\text { Ln (dados) }\end{array}$} & \multicolumn{2}{|c|}{$\begin{array}{c}\text { Modelo Gravitacional } \\
\text { (da Silva e D'Agosto, 2013) }\end{array}$} \\
\hline & Não & não & $\operatorname{sim}$ & $\operatorname{sim}$ & & \\
\hline & \multicolumn{6}{|c|}{ Balanceamento } \\
\hline & Não & $\operatorname{sim}$ & não & $\operatorname{sim}$ & não & Sim \\
\hline $\mathrm{R}^{2}$ (Tijp* $\mathrm{x}$ Tijr $\left.{ }^{* *}\right)$ & 0,46 & 0,81 & 0,20 & 0,87 & 0,37 & 0,78 \\
\hline ID (Tijp x Tijr) & $52,23 \%$ & $26,35 \%$ & $43,59 \%$ & $21,08 \%$ & $45 \%$ & $28 \%$ \\
\hline $\mathrm{R}^{2}$ (Ln Tijp X Ln Tijr) & 0,05 & - & 0,63 & - & - & - \\
\hline
\end{tabular}

*Tijp: são os valores dos fluxos estimados por cada modelo para o ano de 2010;

**Tijr: são os valores dos fluxos reais para o ano de 2010.

As medidas de desempenho utilizadas mostraram que os modelos com RNA que utilizaram como dados de entrada as variáveis de entradas logaritmizadas apresentaram maior valor de coeficiente de determinação e um índice de dissimilaridade menor em relação ao modelo gravitacional. Foi utilizado como variável independente o Logaritmo Natural das variáveis no C-02, pois a rede produziu resultados negativos para a variável de resposta sem essa consideração. Para evitar essa incoerência de resultados, Madalozo, Dyminski e Ribeiro (2004) utilizaram um artifício denominado de clip através do programa computacional MatLABC), cuja função é de automaticamente igualar a zero toda saída negativa. Já no presente artigo, optou-se por utilizar o Logaritmo Natural (ln) para transformar as saídas negativas em um valor próximo de zero, possuindo menor erro se comparado ao artifício clip.

\section{Conclusão}

O trabalho procurou propor um procedimento para a utilização de RNA na distribuição de viagens de carga, afim de mostrar mais uma opção para o cálculo do mesmo, já que a mesma não é evidenciada na literatura como uma alternativa, visando também contribuir para o desenvolvimento de novas técnicas aplicadas no estudo de planejamento de transporte de carga para auxiliar no preenchimento da lacuna na publicação de trabalhos sobre o uso do Método de quatro etapas e RNAs em periódicos científicos.

0 procedimento proposto foi capaz de estimar a matriz O-D de fluxo de soja destinada à exportação no Brasil. A combinação C-02, construída e treinada com o software Mathwoks MatLAB/NNTC, permitiu conhecer a variação de demanda e destacou-se por apresentar maior precisão em relação ao MG, apresentando um coeficiente de determinação $\left(\mathrm{R}^{2}\right)$ superior em 9\% e tendo Índice de Dissimilaridade (ID) 6,92\% menor, mostrando então uma nova alternativa para o cálculo da distribuição de viagens, porém, este procedimento pode ser aplicado para outros produtos. As estimativas, ainda, apresentaram erro que podem ser melhorados em pesquisas futuras com análise de outros algoritmos de aprendizagem e outras funções de ativação.

As estimativas com RNA são melhores do que as com o MG, o que já afirmava Raia Jr (2000) mesmo sendo razoavelmente conhecida em diversos ramos de atividades, somente nesta última década tem sido utilizada com mais frequência na Engenharia de Transportes e parece ser adequada para este tipo de aplicação, pois de acordo com alguns autores, ela tem mostrado um desempenho superior quando comparada com tradicionais técnicas de regressão, principalmente pela sua capacidade de capturar a não linearidade por detrás dos dados, porém, a forma de entrada dos dados influencia o desempenho e a arquitetura da RNA, o que exigiu a avaliação e otimização dos parâmetros na construção e treinamento dos modelos.

Como propostas para estudos futuros, o método pode ser aplicado a outros produtos e verificar o desempenho dos modelos. Poderá variar as funções de ativação e verificar a existência de outras que possam melhorar o desempenho da RNA, como por exemplo a função sigmoidal (Logística) bem como outros outras variações do algoritmo de aprendizado backpropagation, como por exemplo o Resilient backpropagation. Poderão ser utilizadas também outras variáveis com a finalidade de aumentar a capacidade de explicação do modelo. 


\section{Referências}

Aguiar Júnior, S. R. (2004) Modelo RAPIDE: uma aplicação de mineração de dados e redes neurais artificiais para a estimativa da demanda por transporte rodoviário interestadual de passageiros no Brasil. Dissertação (Mestrado em Gestão do conhecimento e da Tecnologia da informação), Universidade Católica de Brasília, Brasília.

Alves, V. F. B., Silva, A. N. R., \& Waerden, P. (2012) Técnicas exploratórias para localizar potenciais usuários de transporte público urbano. Journal of Transport Literature, 6 (3), 180-203.

Araújo, R. B., \& Melo, P. L. S. V. (2006) Protótipo de Software para aprendizagem de Redes Neurais Artificiais. Anaiss do XXXIV COBENGE. Ed. Universidade de Passo Fundo. Passo Fundo.

Batista; B. C. F. (2012) Soluções de Equações Diferenciais usando Redes Neurais de Múltiplas camadas com os métodos da Descida mais íngreme e Levenberg-Marquardt. Dissertação (Mestrado em Matemática), Universidade Federal do Pará, Bélem.

Brondino, M. C. (1999) Estudo da influência da acessibilidade no valor de lotes urbanos através de redes neurais. Tese (Doutorado em Engenharia Civil - Transportes), Escola de Engenharia de São Carlos da Universidade de São Paulo, São Paulo.

Bruton, M. J. (1979) Introdução ao planejamento dos transportes. São Paulo: Editora da universidade de São Paulo.

Confederação Nacional do Transporte - CNT. (Maio de 2012) disponível em www.cnt.org.br

Celik, H. M. (2004) Modeling freight distribution using artificial neural networks. Journal of Transport Geography, 12, $141-148$.

Corrêa, F. (2008) Aplicação de redes neurais artificiais no setor de transportes no Brasil. Dissertação (Mestrado em Engenharia Urbana), Universidade Federal de São Carlos, São Paulo.

da Silva, M., \& D’Agosto, M. (2013) A model to estimate the origin-destination matrix for soybean exportation in Brazil. Journal of Transport Geography, 26, 97-107.

Dia, H. (2001) An object-oriented neural nerwork approach to short-tem traffic forecasting. European Journal of Operational Research, 131, 253-261.

Dougherty, M. S., \& Cobbett, M. R. (1997) Short-term inter-urban traffic forecastsusing neural networks. International Journal of Forecasting,vol. 12, 21-31.

Empresa Brasileira de Pesquisa Agropecuária - EMBRAPA (2011). A soja. Relatório sumário da situação da soja. Disponível em www.embrapa.br.

Kaiser, M. S., Prata, P. A., \& Ribeiro, G. M. (2007) Análise comparativa entre o TransCAD e heurísticas de agrupamento e de roteamento de veículos. XXI Associação Nacional de Pesquisa e Ensino em Tranporte, Rio de Janeiro. Disponível em www.cbtu.gov.br.

Kirby, H. R., Watson, S. M., \& Dougherty, M. S. (1997) Should we use neural networks or statistical models for short-term motorway traffic forecasting?, International Journal of Forecasting, 1 (1), 43-50.

Knudsen, D. C., \& Fotheringham, A. S. (1986) Matrix Comparison, Goodness-of-Fit, and Spatial Interaction Modeling. International Regional Science Review, 10 (2), 127-147.

Lo, H., Zhang, N., \& Lam, W. (1996) Estimation of an origin-destination matrix with random link choice proportions: a statistical approach. Transportation Research Part B,30 (4), 309-324.

Madalozo, H. C. Dyminski, A. S., \& Ribeiro, E. O. (2004) Análise de curvas horizontais de rodovias, para melhoramento de projeto e operação, utilizando redes neurais artificiais. Anais do $18^{\circ}$ Congresso Brasileiro de Transporte e Trânsito -ANPET, Santa Catarina.

Nakatsuji, T.,\& Kaku, T. (1989) Application of Neural Network Models to Traffic Engineering Problems. Proceedings of the Infrastructure Planning, 12, 297-403.

Ortuzar, J. D., \&e Willumsen, L. G. (2011) Modelling Transport - 4th ed. New York: Wiley\&Sons.

Raia Jr, A. A. (2000) Acessibilidade e mobilidade na estimativa de um índice de potencial de viagens utilizando redes neurais artificiais e sistemas de informações geográficas. Tese (Doutorado em Engenharia Civil - Transportes), Escola de Engenharia de São Carlos, Universidade de São Paulo, São Paulo.

Scalea, L. (2002) Transporte e Armazenagem de Soja no Brasil. Rio de Janeiro: Instituto Militar de Engenharia.

Sistema de Análise das Informações de Comércio Exterior via Internet - Alice_Web. (2013) Dados referentes à exportação de soja, disponível em aliceweb.desenvolvimento.gov.br.

Sistema de Informações de Fretes - SIFRECA (2012). Disponível em sifreca.esalq.usp.br.

Sikdar, P. K., \& Hutchinson, B. G. (1981) Empirical Studies of Work Trip Distribution Models. Transportation Research Part A, vol, 15 (3), 233-243.

Smith, D. P. e Hutchinson, G. B. (1981) Goodness of Fit Statistics for trip Distribution Models. Transpotation Research , vol. 15 (4), $295-303$.

Souza, C. D. R., \& D'Agosto, M. A. (2013) Modelo de quatro etapas aplicado ao planejamento de transporte de carga. Journal of Transport Literature, 7 (2), 207-234.

Wilson, A. G. (1970) Entropy in Urban and Regional Modelling. London: Pion.

Wilson, S. R. (1976) Statistical Notes on the Evaluation of Calibrated Gravity Models. Transportation Research, 10 (5), $343-345$.

Yuehua, Z. H. U., Chen, Y., Geng, X., \& Liu, L. (2008) Transport Modal Split of Commercial Sites Based on Artificial Neural Network. Journal of Transportation, 8 (1).

\section{Abstract}

This paper aims to use the technique of artificial neural networks (ANN) to estimate the origin-destination matrix (O-D) of soybeans in Brazil for export to explain the variability of flows between O-D pairs, considering the dynamic characteristics existing in 0 -D matrices. We will then compare the results with the gravity model (GM), which is a model used in strategic planning of the brazilian government also proposed a procedure for the use of anns in travel distribution load. among the four models built in this paper, we have highlighted the combination C-02, with a coefficient of determination $\left(\mathrm{R}^{2}\right.$ ) higher than $9 \%$ and with a dissimilarity index (DI) $6.92 \%$ lower than the GM. It can be observed that anns can be a potential substitute to conventional statistical models because if their easy interface to user programs, but the ANNs are not reported in the literature as options for calculating the travel distribution of Four Steps Method. Thus, this technique is rarely used for the transportation of cargo.

Key words: neural networks, soybean exportation flows, cargo flow prediction, trip distribution 